\title{
Composición y estructura de la ictiofauna demersal en la zona norte de Chile*
}

\author{
Walter Sielfeld K. y Mauricio Vargas F. \\ Departamento de Ciencias del Mar, Universidad Arturo Prat \\ Casilla 121, Iquique, Chile
}

Resumen: Se analiza la composición y estructura de la ictiofauna demersal en la zona norte de Chile (18 $24^{\circ}-21^{\circ} 26^{\prime}$ S $)$. Se registraron 50 especies pertenecientes a 16 órdenes y 31 familias de peces. Se amplía el rango de distribución sur para las siguientes especies: Dibranchus spinosa, Cherublemma emmelas, Pachycara suspectum; y el rango de distribución norte para Centroscyllium granulatum. Las familias mejor representadas son Macrouridae, Ophidiidae y Zoarcidae (6, 5 y 4 especies, respectivamente). Por su abundancia destacan las familias Macrouridae y Ophidiidae (46\% y $21 \%$, respectivamente) y las especies Trachyrhynchus helolepis (33\%), Cherublemma emmelas (21\%) y Trachyrhynchus villegai (8\%). Las especies más frecuentes fueron Coryphaenoides ariommus (69\%) y Trachyrhynchus helolepis (56\%).

El análisis batimétrico indica la existencia de un conjunto demersal somero (0-100 m) y un conjunto demersal profundo (100-1000 m). El estudio de la composición específica permite reconocer la existencia de tres niveles batimétricos en el segundo conjunto (200-500, 500-700 y 700-1000 m). La distribución latitudinal indica que la comunidad representa un estado transicional entre las asociaciones de la plataforma continental y el talud del sector Panamá-Perú y Chile central, dominando elementos del primero.

Palabras claves: demersal, norte de Chile, estructura específica, distribución batimétrica y latitudinal.

\section{Composition and structure of the demersal ichtyofauna in northern Chile*}

\begin{abstract}
The species composition and structure of the demersal ichtyofauna of the northern Chile (18 $24^{\prime}-21^{\circ} 26^{\prime}$ 'S was studied. 50 fish species, belonging to 16 orders and 31 families were recorded. The distribution range of the following species extends south to the study area: Dibranchus spinosa, Cherublemma emmelas, Pachycara suspectum and the northern distribution range of Centroscyllium granulatum. The best represented families were Macrouridae, Ophidiidae and Zoarcidae (6, 5 and 4 species, respectively). The most abundant families were Macrouridae and Ophidiidae (46\% and 21\%, respectively) and the most abundant species Trachyrhynchus helolepis (33\%), Cherublemma emmelas (21\%) and Trachyrhynchus villegai (8\%). The most frequent species were Coryphaenoides ariommus (69\%) and Trachyrhynchus helolepis $(56 \%)$.

The bathymetric distribution shows a shallow-demersal species group (0-100 m) and deep-demersal species group (100-1000 m), the last with three different bathymetric levels: 200-500, 500-700 y 700-1000 m. The latitudinal distribution of the fish species showed the existence of a transition between the fish associations of the continental shelf and slope off Panama-Peruvian and central Chile, belonging the dominant elements to the first one.
\end{abstract}

Key words: demersal, northern Chile, specific composition, bathymetrical and latitudinal distribution.

*Estudio derivado del proyecto PNUD-CHI/87/007 


\section{INTRODUCCION}

Los estudios de las asociaciones ecológicas de peces en Chile has estado referidos principalmente a ambientes submareales e intermareales costeros (Moreno et al., 1979; Núñez y Vásquez, 1987; Vásquez, 1989; Varas y Ojeda, 1990), al ser sistemas asequibles a la observación independiente y directa mediante buceo autónomo (Moreno et al., 1979). El conocimiento que se tiene de las asociaciones ícticas sublitorales es escaso y está relacionado con información derivada de las pesquerías de arrastre existentes desde el sur de Coquimbo hasta la región de Magallanes (Pequeño, 1975; Yáñez y Barbieri, 1983; Ojeda, 1983; Arancibia, 1992). Kong y Meléndez (1991) describieron la ictiofauna de las aguas profundas entre Arica e Isla Mocha con un enfoque sistemático.

Para buscar nuevos recursos hidrobiológicos para el sector pesquero del norte de Chile, en 1988-89 se realizó un estudio de los recursos pesqueros no tradicionales de la zona y sus potencialidades con el proyecto. Aplicación de nuevas tecnologías para capturar recursos hidrobiológicos marinos no tradicionales en la I Región de Chile. Esta actividad se desarrolló con el apoyo del Programa Nacional de Cooperación Técnica del Gobierno de Chile y el respaldo del Programa de las Naciones Unidas para el Desarrollo.

En este trabajo se presentan los resultados sobre la composición específica, estratificación batimétrica y relaciones zoogeográficas de la ictiofauna demersal explorada. Aspectos tales como densidad, biomasa disponible y maduración gonadal de las especies de importancia pesquera, han sido tratados previamente por Claramunt (1993) y Herrera (1993); además, Fuenzalida (1993) discute las condiciones oceanográficas físicas de los lugares de muestreo.

\section{MATERIALES Y METODOS}

\section{Características del área de estudio}

La plataforma continental ubicada entre Arica

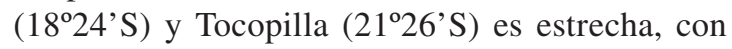
existencia de terrazas formadas por sedimentos aluviales (Fisher y Raitt., 1962; Coulbourn, 1981), y el talud de gran pendiente hacia la fosa ChilenoPeruana. El área de estudio se ubica a $110 \mathrm{~km}$ en los $21^{\circ} \mathrm{S}$ y $170 \mathrm{~km}$ en los $18^{\circ} 30^{\prime} \mathrm{S}$ (Fig. 1). En estos rangos se ubican las mayores profundidades de la zona, alcanzando los $8.000 \mathrm{~m}$ frente a los $23^{\circ} 27^{\prime} \mathrm{S}$ (Schweller et al., 1984).

Según Sievers y Silva $(1975,1982)$, la Corriente de Humboldt se presenta dividida en dos ramas frente a la I Región de Chile, una costera ubicada entre $71^{\circ}$ y $73^{\circ} \mathrm{W}$, y otra oceánica entre $73^{\circ}$ y $77^{\circ} \mathrm{W}$. En consecuencia, el área de estudio queda limitada por el oeste por la rama costera y por el este por la franja de surgencias litorales que caracterizan el norte de Chile (Fuenzalida, 1990).

\section{Sistema de muestreo y arte de pesca}

La prospección se realizó con el B/I "Carlos Porter" del Instituto de Fomento Pesquero, del 3 al 7 de septiembre de 1998 , entre $18^{\circ} 24^{\prime} \mathrm{S}$ y $21^{\circ} 26^{\prime} \mathrm{S}$ y $70^{\circ} 3$ 'W y $72^{\circ} 04^{\prime} \mathrm{W}$ (Fig. 1), realizándose 21 lances de pesca a profundidades entre 34 y $1.050 \mathrm{~m}$ (Tabla1). Se utilizó una red de arrastre de polietileno, tipo camaronera de dos paneles, con abertura de malla de $40 \mathrm{~mm}$, cuyas características fueron: relinga superior $21 \mathrm{~m}$, relinga inferior $25 \mathrm{~m}$, ancho máximo $10 \mathrm{~m}$, largo del ala $7 \mathrm{~m}$, del cielo $7 \mathrm{~m}$ y del copo $6 \mathrm{~m}$ (Palma, 1993). Cada arrastre se realizó a una velocidad de 2 nudos y durante 30 minutos, lo que hace comparable las capturas en términos de abundancia relativa.

Se consideró en este caso como peces demersales a aquellos que dependen del fondo marino en alguna de sus fases de desarrollo y que se les captura mediante un arte de pesca de arrastre, hasta una altura de $20 \mathrm{~m}$ sobre el fondo.

Debido al carácter multiespecífico que tuvo la prospección, y a la falta de información sobre los organismos disponibles al arte de pesca y el tipo de fondo que condiciona la zona, no se pudo estratificar el área antes del crucero. Por lo tanto se optó por el muestreo al azar, con una post-estratificación de la muestra (Palma, 1993), considerándose estratos batimétricos en intervalos de $100 \mathrm{~m}$.

El material obtenido fue separado por especie y fijado en formaldehído al 5\%; luego fue determinado y contabilizado por estación y lance de pesca. Para consultas posteriores éste se conserva en la Colección Zoológica del Departamento de Ciencias del Mar, de la Universidad Arturo Prat de Iquique. 
W

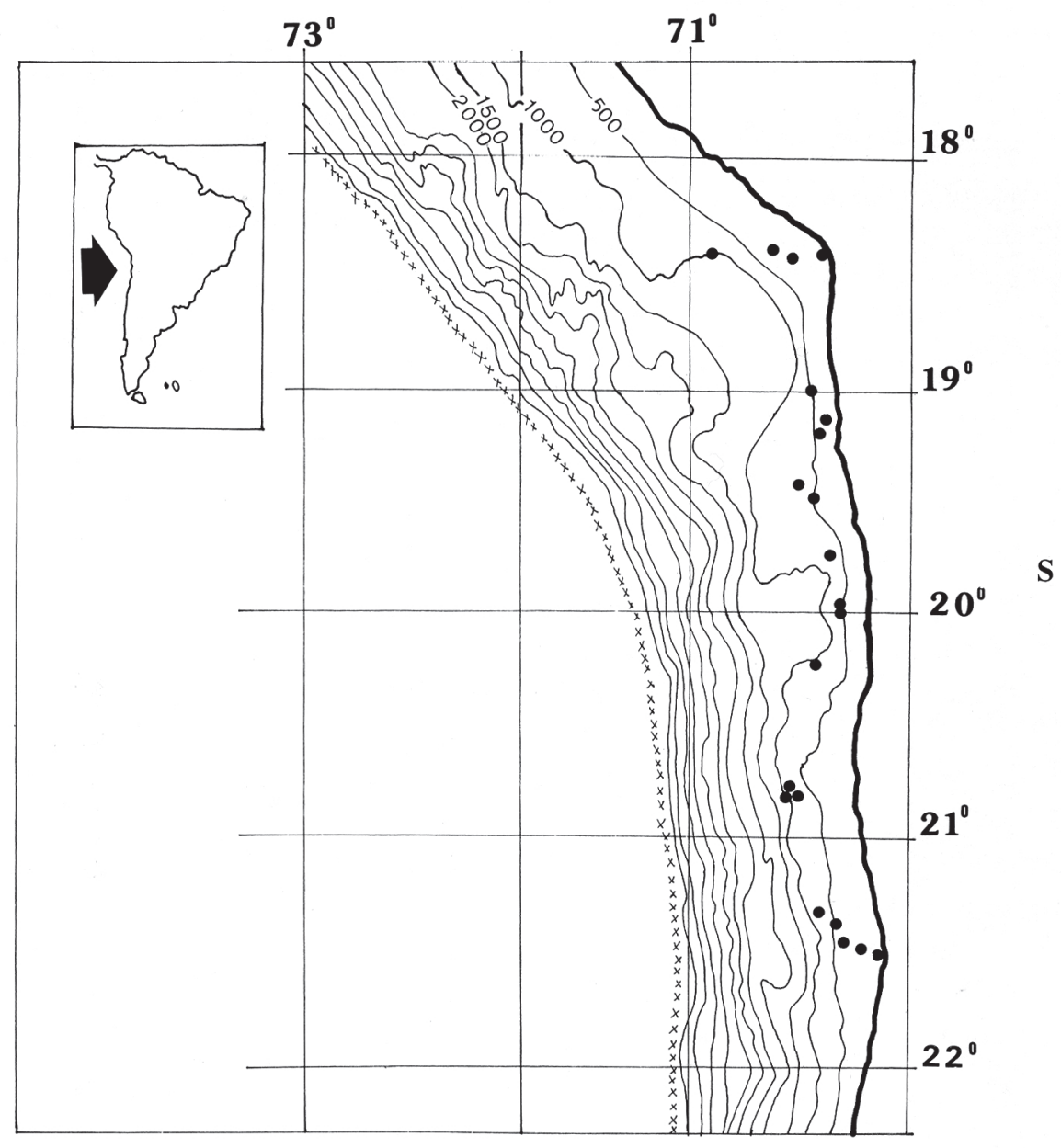

Figura 1. Ubicación de las estaciones de muestreo y batimetría del fondo del área de estudio (Coulbourn, 1981). La línea de cruces indica ubicación del fondo de la fosa chileno-peruana.

Figure 1. Locations of the sampling stations and the bathymetry of the seabed in the study area. The line of crosses indicates the location of the bottom of the Chile-Peru trench.

\section{Clasificación de las colectas}

La identificación de especies y su distribución consultó las siguientes revisiones generales: Günther (1887), Garman (1899), Hildebrand (1946), Mann (1954), De Buen (1961a y b), Cervigón (1972), Chirichigno $(1974,1978)$ y Kong y Meléndez (1991). Para Ophidiidae se consultó a Cohen y Nielsen (1978) y Kong et al. (1988); para Zoarcidae, Anderson y Peden (1988), Anderson (1988) y Pequeño (1986); para Macrouriformes, Iwamoto
(1978, 1979), Iwamoto y Sazonov (1988) y Sazonov e Iwamoto (1992); y para Melamphaeidae, Ebeling (1962) y Ebeling y Weed (1963).

\section{Parámetros y criterios de medida}

Para el análisis de fidelidad se siguió a Sáiz (1975), quien señala las siguientes categorías:

- exclusiva: especie sólo presente en un determinado ambiente. 
Tabla 1. Detalle de las estaciones del muestreo y lances de arrastre. Table 1. Details of the sampling stations and of the trawls.

\begin{tabular}{|c|c|c|c|c|c|c|c|}
\hline $\mathbf{N}^{\circ}$ & Latitud & Longitud & $\begin{array}{c}\text { Profundidad } \\
\text { de inicio }(\mathrm{m})\end{array}$ & $\begin{array}{c}\text { Profundidad } \\
\text { de término }(\mathrm{m})\end{array}$ & $\begin{array}{c}\text { Inicio } \\
(\mathrm{hr})\end{array}$ & $\begin{array}{c}\text { Término } \\
(\mathrm{hr})\end{array}$ & Fecha \\
\hline 01 & $21^{\circ} 25^{\prime}$ & $70^{\circ} 05^{\prime}$ & 34 & 38 & $07: 15$ & $07: 46$ & $08 / 09 / 88$ \\
\hline 02 & $21^{\circ} 25^{\prime}$ & $70^{\circ} 17^{\prime}$ & 200 & 305 & $11: 30$ & $12: 00$ & $08 / 09 / 88$ \\
\hline 03 & $21^{\circ} 20^{\prime}$ & $70^{\circ} 21^{\prime}$ & 520 & 505 & $16: 58$ & $17: 28$ & $08 / 09 / 88$ \\
\hline 04 & $21^{\circ} 10^{\prime}$ & $70^{\circ} 26^{\prime}$ & 605 & 610 & $18: 40$ & $19: 20$ & $08 / 09 / 88$ \\
\hline 05 & $20^{\circ} 46^{\prime}$ & $70^{\circ} 34$ & 700 & 780 & $07: 30$ & $08: 00$ & $08 / 09 / 88$ \\
\hline 06 & $20^{\circ} 47^{\prime}$ & $70^{\circ} 34^{\prime}$ & 1050 & 920 & $11: 00$ & $11: 30$ & $08 / 09 / 88$ \\
\hline 07 & $20^{\circ} 49^{\prime}$ & $70^{\circ} 29^{\prime}$ & 490 & 460 & $14: 20$ & $14: 50$ & $09 / 09 / 88$ \\
\hline 08 & $20^{\circ} 11^{\prime}$ & $70^{\circ} 19^{\prime}$ & 705 & 740 & $07: 05$ & $07: 35$ & $09 / 09 / 88$ \\
\hline 09 & $19^{\circ} 58^{\prime}$ & $70^{\circ} 17^{\prime}$ & 470 & 440 & $11: 26$ & $12: 18$ & $09 / 09 / 88$ \\
\hline 10 & $19^{\circ} 53^{\prime \prime}$ & $70^{\circ} 15^{\prime}$ & 480 & 470 & $15: 20$ & $15: 50$ & $10 / 09 / 88$ \\
\hline 11 & $19^{\circ} 45^{\prime}$ & $70^{\circ} 17^{\prime}$ & 700 & 680 & $18: 40$ & $19: 15$ & $10 / 09 / 88$ \\
\hline 12 & $19^{\circ} 31^{\prime}$ & $70^{\circ} 23^{\prime}$ & 470 & 520 & $09: 35$ & $10: 05$ & $10 / 09 / 88$ \\
\hline 13 & $19^{\circ} 28^{\prime}$ & $70^{\circ} 24^{\prime}$ & 700 & 700 & $10: 45$ & $11: 15$ & $10 / 09 / 88$ \\
\hline 14 & $19^{\circ} 12^{\prime}$ & $70^{\circ} 20^{\prime}$ & 390 & 170 & $15: 25$ & $15: 55$ & $11 / 09 / 88$ \\
\hline 15 & $18^{\circ} 25^{\prime}$ & $70^{\circ} 45^{\prime}$ & 500 & 280 & $07: 25$ & $08: 55$ & $11 / 09 / 88$ \\
\hline 16 & $18^{\circ} 24^{\prime}$ & $70^{\circ} 20^{\prime}$ & 50 & 29 & $15: 25$ & $15: 55$ & $12 / 09 / 88$ \\
\hline
\end{tabular}

- preferente: especie netamente más abundante en un determinado ambiente.

- subferente: especie más abundante en otros ambientes.

- indiferente: especie igualmente abundante en diversos ambientes.

- accidental: especie muy escasa en todos los ambientes.

La dominancia numérica y constancia fueron valoradas según el esquema propuesto pos Bodenheimer (1955 fide Domínguez y Zúñiga, 1976). El índice de constancia se refiere al número de presencias en el total de las recolecciones, se expresa en porcentaje y califica las especies en las siguientes categorías según su incidencia (Solervicens, 1973):

- especie constante con $50 \%$ o más de presencia,

- especie accesoria con $25-50 \%$ de presencia, y

- especie accidental con 10-25\% de presencia.

Como especie dominante se define aquella cuya abundancia relativa es superior al 5\% (Solervicens, 1973), donde la abundancia relativa representa el número relativo de individuos de una especie en comparación al total de todas las especies.

La diversidad específica se obtuvo el índice de diversidad de Shannon-Wiener (H'), por constituir una medida clásica en estudios de tipo comunitario. La expresión utilizada fue:

$$
\mathrm{H}^{\prime}=\mathrm{p}_{\mathrm{i}} \log _{2} \mathrm{p}_{\mathrm{i}}
$$

donde:

$\mathrm{p}_{\mathrm{i}}=\mathrm{n}_{\mathrm{i}} / \mathrm{N}$; proporción del número de ejemplares en la i-ésima especie.

Para estimar la similitud se utilizó el análisis de "clusters", expresado en forma de dendrogamas a partir del índice de similitud de Jaccard $\left(\mathrm{S}_{\mathrm{j}}\right)$. La estrategia aglomerativa utilizada fue el método del promedio aritmético no ponderado (Menni y Goztonyi, 1982). Los datos fueron ingresados al programa computacional ACOM (Navarro, 1984). En la elección del índice se siguió a Sáiz (1980) y en la determinación del nivel de similitud a Arancibia (1988). La expresión utilizada fue: 


$$
\mathrm{S}_{\mathrm{i}}=\frac{\mathrm{a}}{\mathrm{a}+\mathrm{b}+\mathrm{c}}
$$

donde:

$\mathrm{a}=$ número de especies propias de la muestra a.

$\mathrm{b}=$ número de especies propias de la muestra $\mathrm{b}$.

$\mathrm{c}=$ número de especies comunes entre las muestras a y b.

\section{RESULTADOS}

\section{Consideraciones generales}

Se registraron 50 peces, pertenecientes a 16 órdenes y 31 familias; 41 especies y 25 familias correspondieron a teleósteos, y 9 especies y 6 familias a condrictios (Anexo 1). El número de especies por estación varió entre 14 (estación 11) y 2 (estación 8) (Fig. 2).

Este conjunto incluyó especies típicamente mesopelágicas, tal vez capturadas durante el ascenso de la red, correspondiendo a Cyclothone acclinidens Garman, 1899; Lampanyctus achirus Andriashev, 1962; Triphothurus mexicanus (Gilbert, 1890); y Scopelengys tristis Alcock, 1890.

Las familias mejor representadas numéricamente (Anexo 1), fueron: Macrouridae, Ophidiidae y Zoarcidae con 6, 5 y 4 especies, respectivamente. Como dominantes destacaron las familias Macrouridae (46\%), Orphidiidae (21\%) y Squalidae (12\%); y las especies Trachyrhynchus helolepis Gilbert, 1891 (33\%); Cherublemma emmelas (Gilbert, 1890) (21\%); Centroscyllium granulatum Günther, 1887 (8\%); Isacia conceptionis (Cuvier, 1830) (8\%); y Merluccius gayi (Guichenot, 1848) (6\%) (Tabla 2). Las especies constantes fueron: Coryphaenoides ariommus Gilbert y Thomson, 1916 (69\%) y Trachyrhynchus helolepis Gilbert, 1891 (56\%). Como especies accesorias destacaron Xenomystax atrarius Gilbert, 1891 (38\%); Centroscyllium granulatum Günther, 1889 (25\%); Cephalarus sp. (25\%); Halosaurus radiatus Garman, 1899 (25\%); Cherublemma emmelas (Gilbert, 1890) (25\%); y Scopelogadus mizolepis (Günther, 1878) (25\%).

La abundancia numérica por estación fue variable, no mostrando relación con la profundidad, alcanzando un máximo de $68 \%$ en la estación 15 y menos del $10 \%$ en las otras estaciones (Fig. 3). Según la diversidad por estación (Fig. 4), la comuni- dad presenta su mayor expresión en los estratos más profundos (500-1050 m; estaciones 3, 5 y 6).

\section{Estratificación batimétrica}

El análisis de la distribución batimétrica de las especies (Fig. 5), señala la existencia de dos grupos característicos (similitud menor a 0,1 ), para los cuales se propone los nombres de demersal somero y demersal profundo. El nivel de separación entre éstos es el rango 50-170 m de profundidad, donde en los $50 \mathrm{~m}$ Seriolella violacea Guichenot, 1848; Aphos porosus (Valenciennes, 1837); y Myliobatis peruvianus Garman, 1913, constituyen el piso del grupo de especies demersales someras. En cambio Cherublemma emmelas (Gilbert, 1890); Trachichthys mento Garman, 1899; y Cataetyx simus Garman, 1899, constituyen el techo del grupo de especies demersales profundas. Merluccius gayi (Guichenot, 1848) fue encontrada a $29 \mathrm{~m}$ de profundidad (estación 16), y traspasa la barrera anterior.

La composición específica exhibe los siguientes grupos:

Conjunto de especies demersales someras: Este grupo está representado en el estrato 0-100 m (Fig.6), caracterizado por la presencia de las siguientes especies exclusivas: Seriolella violacea; Aphos porosus; Sciaena deliciosa (Tschudi, 1844); Isacia conceptionis; Paralabrax humeralis (Valenciennes, 1828); Normanichthys crockeri Clark, 1937; Hippoglossina macrops Steindachner, 1876; Myliobatis peruvianus Garman, 1913; y Sympterygia brevicaudata (Cope, 1877), Merluccius gayi, que integra este conjunto, lo hace en forma subferente (2 especímenes en estación 6), al ser más abundante y preferente (188 especímenes) en el piso batimétrico siguiente $(280-500 \mathrm{~m})$. Estas especies, salvo M. gayi, no fueron capturadas bajo $50 \mathrm{~m}$ de profundidad; en consecuencia, estuvieron asociadas a una mezcla de aguas subtropicales y subantárticas (Fuenzalida, 1993).

Conjunto de especies demersales profundas: Incluye las que fueron capturadas sobre el talud, entre 170 m (estación 14) y 1.050 m (estación 6) de profundidad. El análisis de similitud muestra una marcada estratificación (Fig. 5), incluyendo los siguientes subconjuntos batimétricos: 200-500, 500-700 y 700-1000 (similitud menor a 48\%). Las especies co- 


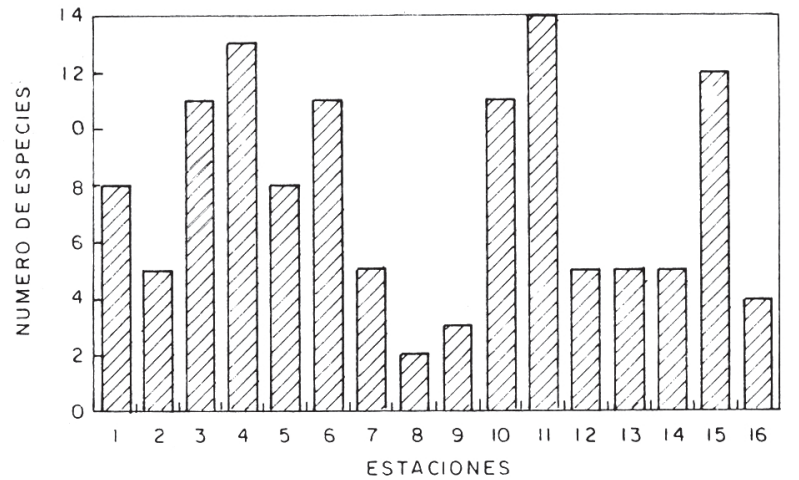

Figura 2. Número de especies por estación de muestreo. Figure 2. Number of species at each sampling station.

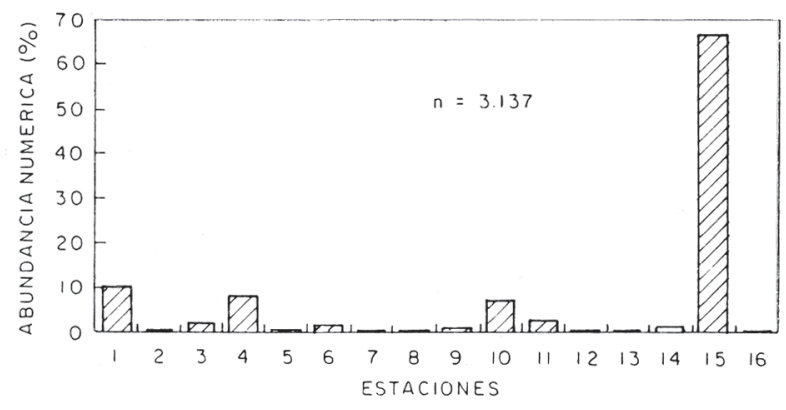

Figura 3. Porcentaje del número total de individuos por estación de muestreo ( $\mathrm{N}=$ número de individuos capturados).

Figure 3. Percentage of the total number of individuals at each sampling station ( $N=$ number of individuals captured).

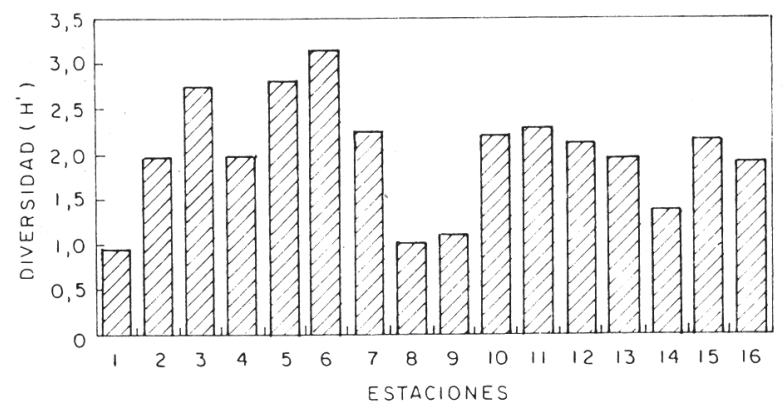

Figura 4. Diversidad específica según índice de Shannon-Wiener por estación de muestreo.

Figure 4. Species diversity using the Shannon-Wiener index, at each sampling station. munes en los tres estratos son Trachyrhynchus helolepis, Xenomystax atrarius y Coryphaenoides ariommus, cuyas capturas fueron más abundantes en las profundidades intermedias (450-700 m).

El estrato 200-500 m se caracterizó por las especies exclusivas Aculeola nigra De Buen, 1959; Ophichthus ater Peters, 1866; Pachychara suspectum (Garman, 1899); y Cherublemma emmelas. El estrato 500-700 m se caracterizó por las especies exclusivas Bathyraja peruana McEachran y Miyake, 1984; Melamphaes acanthomus Ebeling, 1962; Psychrolutes sio Nelson, 1980 y Cataetyx rubrirostris Gilbert, 1890.

En el estrato 700-1000 m se capturó Coryphaenoides anguliceps (Garman, 1899); Roulenia sp.; Bothrocara alalonga (Garman, 1899) y Pachychara sp. A diferencia de los estratos anteriores, éste no presentó condrictios.

El estrato 100-200 m destaca como unidad aislada (similitud 0,07) (Fig. 5), lo que deriva de su pobreza en especies (Merluccius gayi, Cherublemma emmelas, Trachichthys mento y Cataetyx simus). Siendo éstas comunes a los estratos más profundos, se les considera como límite superior del conjunto de especies demersales profundas.

\section{Aspectos zoogeográficos}

Las especies demersales someras son propias de las zooprovincias centro-chilena y peruana (sensu Balech (1954), y Pequeño y Lamilla (1993)); no sobrepasan el Ecuador, salvo Hippoglossina macrops que alcanza las costas mexicanas (Chirichigno, 1974).

La distribución geográfica conocida para el conjunto de especies demersales profundas (Fig. 6), señala la presencia de los siguientes conjuntos característicos:

Elementos peruano-panameños: son características de la plataforma y/o talud frente a Panamá-Perú (Anexo1), y corresponden a Cephalurus sp.; Centroscyllium nigrum Garman, 1889; Bathyraja peruana, 1984; Gurgesiella furvescens De Buen, 1959; Halosaurus radiatus Garman, 1899; Xenomystax atarius; Dibranchus spinosa (Garman, 1899); Coryphaenoides delsolari Chirichigno y 
Tabla 2. Abundancia relativa, frecuencia de aparición y rangos batimétricos de las especies demersales capturadas.

Table 2. Relative abundance, frequency of occurrence, and bathymetric ranges of the demersal species captured.

\begin{tabular}{|c|c|c|c|c|c|}
\hline Especies & \multicolumn{2}{|c|}{ Abundancia } & Frecuencia & $\begin{array}{l}\text { uencia } \\
(\%)\end{array}$ & $\begin{array}{c}\text { Rango } \\
\text { batimétrico (m) }\end{array}$ \\
\hline Aculeola nigra & 116 & $(3,7)$ & 3 & $(18,8)$ & $280-520$ \\
\hline Centroscyllium granulatum & 259 & $(8,3)$ & 4 & $(25,0)$ & $280-610$ \\
\hline Centroscyllium nigrum & 12 & $(0,4)$ & 3 & $(18,8)$ & $280-610$ \\
\hline Cephalurus sp. & 11 & $(0,4)$ & 4 & $(25,0)$ & $505-700$ \\
\hline Bathyraja peruana & 1 & $(<0,1)$ & 1 & $(6,3)$ & $680-700$ \\
\hline Sympterygia brevicaudata & 1 & $(<0,1)$ & 1 & $(6,3)$ & $34-38$ \\
\hline Gurgesiella furvescens & 1 & $(<0,1)$ & 1 & $(6,3)$ & $605-610$ \\
\hline Myliobatis peruvianus & 2 & $(0,1)$ & 2 & $(12,5)$ & $34-50$ \\
\hline Hydrolagus macrophthalmus & 3 & $(0,1)$ & 2 & $(12,5)$ & $680-780$ \\
\hline Xenomystax atrarius & 60 & $(1,9)$ & 6 & $(37,5)$ & $280-1050$ \\
\hline Ophicthus ater & 1 & $(<0,1)$ & 1 & $(6,3)$ & $280-500$ \\
\hline Nemichthys scolopaceus & 1 & $(<0,1)$ & 1 & $(6,3)$ & $700-780$ \\
\hline Notacanthus sexpinnis & 4 & $(0,1)$ & 2 & $(12,5)$ & $470-700$ \\
\hline Halosaurus radiatus & 5 & $(0,2)$ & 4 & $(25,0)$ & $280-780$ \\
\hline Cyclothone acclinidens & 3 & $(0,1)$ & 1 & $(6,3)$ & $440-470$ \\
\hline Alepocephalus sp. & 7 & $(0,2)$ & 3 & $(18,8)$ & $470-780$ \\
\hline Binghamichthys microphos & 5 & $(0,2)$ & 2 & $(12,5)$ & $605-1050$ \\
\hline Roulenia sp. & 2 & $(0,1)$ & 1 & $(6,3)$ & $920-1050$ \\
\hline Lampanyctus achirus & 4 & $(0,1)$ & 3 & $(18,8)$ & $440-780$ \\
\hline Triphoturus mexicanus & 1 & $(<0,1)$ & 1 & $(6,3)$ & $505-520$ \\
\hline Scopelengys tristis & 6 & $(0,2)$ & 3 & $(18,8)$ & $470-740$ \\
\hline Aphos porosus & 4 & $(0,1)$ & 2 & $(12,5)$ & $29-50$ \\
\hline Dibranchus spinosa & 3 & $(0,1)$ & 2 & $(12,5)$ & $680-1050$ \\
\hline Merluccius gayi & 190 & $(6,1)$ & 6 & $(37,5)$ & $29-520$ \\
\hline Coryphaenoides ariommus & 118 & $(3,8)$ & 11 & $(68,8)$ & $280-1050$ \\
\hline Coryphaenoides delsolari & 4 & $(0,1)$ & 3 & $(18,8)$ & $460-610$ \\
\hline Coryphaenoides anguliceps & 1 & $(<0,1)$ & 1 & $(6,3)$ & $920-1050$ \\
\hline Nezumia pulchella & 9 & $(0,3)$ & 3 & $(18,8)$ & $280-520$ \\
\hline Trachyrhynchus helolepis & 1028 & $(32,8)$ & 9 & $(56,3)$ & $280-1050$ \\
\hline Trachyrhynchus villegai & 275 & $(8,8)$ & 5 & $(31,3)$ & $280-700$ \\
\hline Cherublemma emmelas & 644 & $(20,5)$ & 4 & $(25,0)$ & $70-500$ \\
\hline Dicrolene nigra & 5 & $(0,2)$ & 2 & $(12,5)$ & $680-1050$ \\
\hline Monomitopus torvus & 3 & $(0,1)$ & 2 & $(12,5)$ & $680-1050$ \\
\hline Cataetyx rubrirostris & 3 & $(0,1)$ & 3 & $(18,8)$ & $280-700$ \\
\hline Cataetyx simus & 1 & $(<0,1)$ & 1 & $(6,3)$ & $170-320$ \\
\hline Bothrocara alalonga & 1 & $(<0,1)$ & 1 & $(6,3)$ & $920-1050$ \\
\hline Pachychara suspectum & 2 & $(0,1)$ & 1 & $(6,3)$ & $280-500$ \\
\hline Pachycharasp. & 1 & $(<0,1)$ & 1 & $(6,3)$ & $920-1050$ \\
\hline Zoarcidae indet. & 1 & $(<0,1)$ & 1 & $(6,3)$ & $470-520$ \\
\hline Melamphaes acanthomus & 1 & $(<0,1)$ & 1 & $(6,3)$ & $680-700$ \\
\hline Scopelogadus mizolepis & 13 & $(0,4)$ & 4 & $(25,0)$ & $440-780$ \\
\hline Trachichthys mento & 10 & $(0,3)$ & 1 & $(6,3)$ & $170-390$ \\
\hline Psychrolutes sio & 1 & $(<0,1)$ & 1 & $(6,3)$ & $680-700$ \\
\hline Normanichthys crockeri & 2 & $(0,1)$ & 1 & $(6,3)$ & $34-38$ \\
\hline Paralabrax humeralis & 1 & $(<0,1)$ & 1 & $(6,3)$ & $34-38$ \\
\hline Isacia conceptionis & 246 & $(7,8)$ & 1 & $(6,3)$ & $34-38$ \\
\hline Sciaena deliciosa & 1 & $(<0,1)$ & 1 & $(6,3)$ & $34-38$ \\
\hline Aphanopus carbo & 2 & $(0,1)$ & 2 & $(12,5)$ & $505-700$ \\
\hline Seriolella violacea & 1 & $(<0,1)$ & 1 & $(6,3)$ & $29-50$ \\
\hline Hippoglossina macrops & 61 & $(1,9)$ & 1 & $(6,3)$ & $34-38$ \\
\hline
\end{tabular}




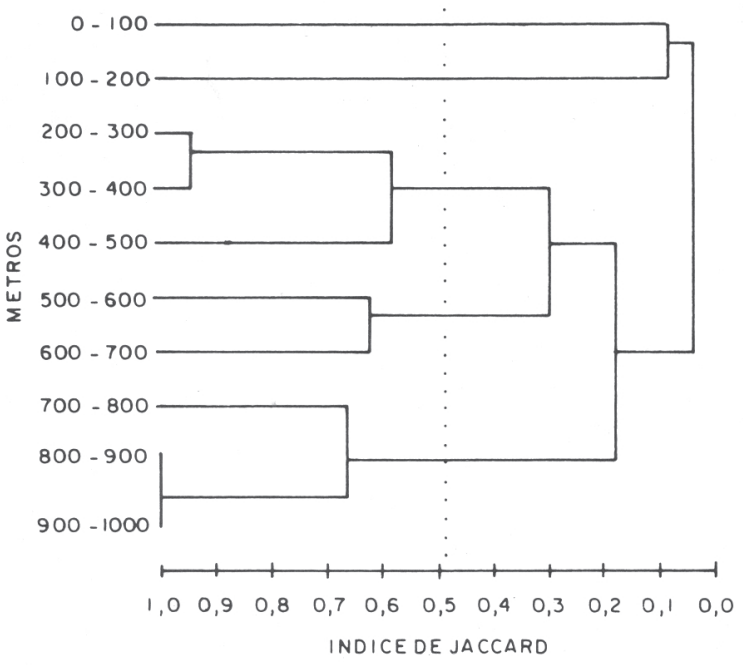

Figura 5. Similitud taxocenótica mediante el índice de Jaccard de la ictiofauna demersal entre estratos batimétricos. La línea de puntos indica el nivel de similitud $(0,48)$.

Figure 5. Taxocenotic similarity, using the Jaccard index, of the demersal ichthyofauna between bathymetric strata. The dotted line indicates the level of similarity $(0,48)$.

Iwamoto, 1977; Coryphaenoides anguliceps; Trachyrhynchus helolepis Gilbert, 1891; Bothrocara alalonga (Garman, 1899); Pachychara suspectum (Garman, 1899); Cherublemma emmelas (Gilbert, 1890); Cataetyx rubrirostris Gilbert, 1890; Cataetyx simus Garman, 1899; Monomitopus torvus Garman, 1899; Trachychthys mento Garman, 1899; y Melamphaes acanthomus Ebeling, 1962. El límite distribucional sur de la mayoría de estas especies calza en el rango $26^{\circ}-35^{\circ} \mathrm{S}$ (Fig. 6), que muestra concordancia con el límite sur de la zooprovincia peruana, indicado por Pequeño y Lamilla (1993) para peces batoideos.

Las especies que en el área registran su límite austral de distribución son (Fig. 6): Bathyraja peruana; Bothrocara alalonga (Garman, 1899); Cherublemma emmelas (Gilbert, 1890); Pachychara suspectum (Garman, 1899); y Dibranchus spinosa (Garman, 1899).

Especies centro-chilenas: son típicas de la plataforma y/o talud de Chile central (Chañaral-Valdivia) (Anexo 1), cuyas distribuciones (Fig. 6) se extienden entre los $2^{\circ} \mathrm{S}$ frente a Perú y los $40^{\circ} \mathrm{S}$ frente a Chile. Corresponden a esta categoría Aculeola nigra De Buen, 1959; Hydrolagus macrophthalmus De
Buen, 1959; Coryphaenoides ariommus, Coryphaenoides anguliceps, Nezumia pulchella (Pequeño, 1971); Trachyrhynchus Villegai Pequeño, 1971; y Psychrolutes sio Nelson, 1980. Con reserva también calza en esta categoría Merluccius gayi, aún cuando su área de distribución es más amplia, desde Galápagos hasta $47^{\circ} \mathrm{S}$ (Martínez, 1976). Este conjunto no es equivalente a la zooprovincia centrochilena (sensu Balech (1954)), al haber sido definida para especies litorales.

Especies de amplia distribución: son circumglobales, con poblaciones en todos los mares tales como Nemichthys scolopaceus Richardson, 1848; Notacanthus sexpinnis Richardson, 1846; Aphanopus carbo Lowe, 1839; y Scopelogadus mizolepis (Günther, 1878).

Especies australes: son características del sector austral (patagónico/magallánico) tales como Centroscyllium granulatum, especie típicamente austral (Meléndez y Meneses, 1989).

\section{DISCUSION}

Sobre la plataforma continental y el talud del norte grande de Chile se encontró una asociación íctica integrada por 50 peces demersales. Esta resulta comparativamente superior a la encontrada en prospecciones realizadas frente al litoral austral de Chile (52-57’S). En efecto, Ojeda (1983) reportó para esta zona 39 especies entre 123 y $600 \mathrm{~m}$ de profundidad. Del mismo modo, los presentes registros contrastan con las 33 especies reportadas por Pequeño (1975) para el sector entre Coquimbo y Corral (30$40^{\circ} \mathrm{S}$ ). Debe considerarse, sin embargo, que en ese caso la mayoría de las capturas fueron realizadas entre 100 y $325 \mathrm{~m}$ de profundidad, rango al que corresponde el conjunto de especies demersales someras del presente estudio.

En términos comparativos, caracterizan el conjunto de peces del litoral de Chile central que describe Pequeño (1975), la notable cantidad de condrictios, a saber: Schroederichthys bivius (Smith, 1841); Schroederichthys chilensis (Guichenot, 1848); Squalus fernandinus Molina, 1782; Discopyge tschudi Heckel, 1841; Raja chilensis Guichenot, 1848; Psammobatis scobina (Philippi, 1892); Symptergia lima (Pöeppig, 1835); y Callorhynchus callorhynchus Linnaeus, 1758; en el 


\begin{tabular}{|c|c|c|}
\hline \multicolumn{2}{|c|}{ ESPECIES } & 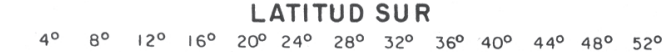 \\
\hline \multirow{29}{*}{$\begin{array}{l}n \\
4 \\
0 \\
2 \\
2 \\
4 \\
0 \\
0 \\
a \\
a\end{array}$} & \multirow{8}{*}{$\begin{array}{l}\text { Notoconthus sexpinnis } \\
\text { Nemichthys scolopaceus } \\
\text { Merluccius gayi } \\
\text { Centroscyillum nigrum } \\
\text { Trochychthys mento } \\
\text { Cotoetyx rubrirostris } \\
\text { Coryphaenoides delsolori } \\
\text { Cotoetyx simus } \\
\text { Halosourus rodiotus }\end{array}$} & 11111 \\
\hline & & 111111 \\
\hline & & $\|1\| 1 \mid$ \\
\hline & & $|1| 1 \mid$ \\
\hline & & $\|1\| \|$ \\
\hline & & $\|1\| \|$ \\
\hline & & $\|[\|||$ \\
\hline & & IIIII \\
\hline & \multirow{9}{*}{$\begin{array}{l}\text { Scopelogodus myzolepis } \\
\text { Monomitopus torvus } \\
\text { Xenomystax atrarius } \\
\text { Gurgesiello furvescens } \\
\text { Cephoturus sp. } \\
\text { Dicrolene nigra } \\
\text { Coryphoenoides ongulice ps } \\
\text { Trochyrhynchus helolepis } \\
\text { Bothyroja peruona }\end{array}$} & 111.11 \\
\hline & & 11111 \\
\hline & & $\$ 111$ \\
\hline & & $|1|||$ \\
\hline & & $\|\Pi\| \|$ \\
\hline & & 11111 \\
\hline & & 11111 \\
\hline & & $\|1\| 11$ \\
\hline & & $\| I I \mid$ \\
\hline & Cherublemmo emmelas & $\|1\|$ \\
\hline & Bothrocara alolonga & 1111 \\
\hline & Melomphoes aconthomus & 1111 \\
\hline & Pochycara suspectum & 111 \\
\hline & Dibranchus spinoso & IIII \\
\hline & Ophichthus ater & III \\
\hline & Psychrolutes s/o & IIIII \\
\hline & Trochyrhynchus villegai & $111 \| 1$ \\
\hline & Hydrolagus macrophtholmus & 11111 \\
\hline & Nezumia puichella & IIIII \\
\hline & Aculeole nigra & 11111 \\
\hline & Coryphaenoides ariommus & 11111 \\
\hline & Centroscyllium granulatum & IIII \\
\hline \multirow{9}{*}{ 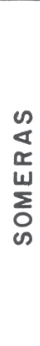 } & Seriolella violoceo & 11111 \\
\hline & Aphos porosus & 11111 \\
\hline & Myliobatis peruvianus & 11111 \\
\hline & Sciaena delicioso & 11111 \\
\hline & Is ocio conceptionis & |IIII \\
\hline & Paralobrox humeralis & 11111 \\
\hline & Normanichthys crockeri & $\| 1111$ \\
\hline & Hippoglossinomacrops & 11111 \\
\hline & & |1111| \\
\hline
\end{tabular}

Figura 6. Distribución geográfica de las especies de peces demersales en la zona de estudio. Las líneas verticales representan el área de estudio y las horizontales la distribución latitudinal conocida de las especies estudiadas.

Figure 6. Geographic distribution of the species of demersal fish in the study area. The vertical lines show the area of study and the horizontal the currently known latitudinal distribution of the species studied. 
norte de Chile, según el presente estudio, se reducen a Sympterygia brevicaudata (Cope, 1877) y Myliobatis peruvians Garman, 1913.

La diversidad media observada $\left(H^{\prime}=2\right)$, se ajusta a los rangos señalados por Targett (1981) para comunidades ícticas tropicales $\left(\mathrm{H}^{\prime}=2-3\right)$ y de la Antártica $\left(H^{\prime}=1,3\right)$, gradiente latitudinal que se reafirma con lo registrado por Arancibia (1992) para comunidades ícticas demersales de Chile central $\left(H^{\prime}=1,6\right)$. Por su parte, la comunidad íctica demersal del presente estudio es más diversa que la comunidad de peces mesopelágicos $\left(H^{\prime}=1,5\right)$, en la misma área del norte de Chile (Sielfeld et al., 1995).

La separación de peces demersales someros del conjunto de peces demersales profundos, en los 50$170 \mathrm{~m}$ de profundidad y de muy baja similitud, probablemente tiene relación con condiciones de temperatura y oxígeno disuelto. Estas son determinadas por la presencia de la corriente de Günther que fluye de norte a sur a lo largo del talud desde $8^{\circ}$ a $45^{\circ} \mathrm{S}$, a profundidades entre 100 y $300 \mathrm{~m}$, con aguas de alta salinidad y escaso contenido de oxígeno (Nekrasov, 1994).

Ante las dificultades técnicas que conlleva la realización de la pesca de arrastre en sectores de profundidades menores a $150 \mathrm{~m}$ y que derivan principalmente de la conformación rocosa del fondo en la zona de estudio, se considera al conjunto de especies demersales someras como submuestreado. Este conjunto debe incluir una serie de especies litorales no detectadas en este estudio, las cuales según lo indicado por diversos autores son típicas del litoral arenoso y rocoso de la zona norte (Günther, 1887; Garman, 1899; Hildebrand, 1946; Mann, 1954; De Buen, 1961a y b; Cervigón, 1972; Chirichigno, 1974, 1978; Kong y Meléndez, 1991).

El análisis de la distribución del conjunto de peces demersales indica un estado de transición entre el conjunto íctico centro-chileno y el conjunto panameño-peruano (Balech, 1954; Pequeño y Lamilla, 1993). La zona de estudio representa el límite de distribución norte del primer conjunto y el sur del segundo. La consideración de las especies señaladas como características en este estudio (especies dominantes, constantes y accesorias), y sus distribuciones (Chirichigno, 1974, 1978; Hildebrand, 1946; McEachran y Miyake, 1984), indican que, a pesar del señalado estado de transición, el conjunto presenta un marcado carácter panameño-peruano.
Las siguientes especies merecen comentarios distribucionales: Dibranchus spinosa, previamente solo detectada hasta Arica, es encontrada en este caso hasta los $20^{\circ} 47^{\prime} \mathrm{S}$ (estaciones 6 y 11 ) Coryphaenoides anguliceps es registrado frente a los 266'S (estación 6); Cherublemma emmelas, conocida anteriormente hasta los $19^{\circ} 19^{\prime} \mathrm{S}$ (Kong et al., 1988), es capturada en $21^{\circ} 25^{\prime}$ 'S (estación 2); Pachychara suspectum conocida hasta Panamá (Anderson y Peden, 1988), es capturada frente a 19¹2’S (estación 15); Meléndez y Meneses (1989) señalan a Centroscyllium granulatum desde el extremo austral hasta los $30^{\circ} 03^{\prime} \mathrm{S}$, rango que es ampliado con el presente trabajo hasta los $18^{\circ} 25^{\prime} \mathrm{S}$ (estación 15); Cephalurus sp. Estuvo representado por especimenes con caracteres claramente genéricos, pero según lo señalado por Kato et al. (1967). Springer (1979) y Compango (1984a) para poblaciones entre Panamá y Chile, difieren de la especie típica por la cabeza significativamente más angosta. Por último, Bathyraja peruana tiene su límite distribucional sur en la zona de estudio, aspecto previamente tratado por Vargas y Sielfeld (1992).

\section{AGRADECIMIENTOS}

Los autores expresan sus agradecimientos al Programa de las Naciones Unidas para el Desarrollo, que hizo posible la realización del proyecto del cual forma parte el material analizado; al Sr. Winston Palma S., Cordinador del Proyecto; y a la valiosa colaboración de tres correctores anónimos, cuyas sugerencias hicieron posible la preparación de la versión final del presente trabajo.

\section{REFERENCIAS}

Anderson, M. 1988. Studies on the Zoarcidae (Teleostei, Perciformes) of the Southern Hemisphere: Two new genera and a new species from temperate South America. Proc. Calif. Acad. Sci., 45(11): 267-276.

Anderson, M. y A. Peden. 1988. The Eelpot Genus Pachycara (Teleostei, Zoarcidae) in the Northeastern Pacific Ocean, with descriptions of two new species. Proc. Calif. Acad. Sci., 46(3): 8394.

Arancibia, H. 1988. Cluster analysis: the use of a simple statistical method in the identification of groups. ICES, CM/D-12 Statistics Cttee., Ref. Demer. Fish 
Cttee., 18 p.

Arancibia, H. 1992. Distribution patterns of the demersal fish assemblage off Central Chile. Biol. Pesq., 21: 43-53.

Balech, E. 1954. Divisiones zoogeográficas del litoral sudamericano. Rev. Biol. Mar., Valparaíso, 4(1-7): 184-195.

Bussing, W. 1965. Studies of the midwater fishes of the Perú-Chile. Trench, biology of the Antarctic seas. Ant. Res. Ser., 5: 185-227.

Cervigón, F. 1972. Los Peces. In: Ecología Marina. Fundación La Salle (Eds.), Dossat, Caracas, 308355.

Chirichigno, N. 1974. Clave para identificar los peces marinos del Perú. Inf. Inst. Mar Perú, Callao, 44: 1-387.

Chirichigno, N. 1978. Nuevas adiciones a la ictiofauna marina del Perú. Inf. Inst. Mar. Perú, Callao, 46: 3109.

Chirichigno, N. y T. Iwamoto. 1977. Coryphaenoides delsolari, a new species of macrourid fish from the Pacific coast of South America. Proc. Biol. Soc. Washington, 89(45): 519-528.

Claramunt, G. 1993. Prospección de recursos bentodemersales: abundancia y análisis de las capturas. In: Aplicación de nuevas tecnologías para la captura de recursos hidrobiológicos marinos no tradicionales en la I Región. W. Palma (Ed.), Proyecto $\mathrm{CHI} / 87 / 007$, PNUD/UNAP, Iquique, Chile, 120 p.

Cohen, D. y J. Nielsen. 1978. Guide to the identification of genera of the fish Order Opidiiformes with a tentative classification of the Order. NOAA Tech. Rep. NMFS, Circ., 417: 1-68.

Compagno, L. 1984a. Sharks of the world. Part 2. Carcharhiniformes FAO Species Catalogue, 4, FIR/ 125: 1-630.

Compagno, L. 1984b. Sharks of the world. Part 1. Hexanchiformes to Lamniformes. FAO Species Catalogue, 4, FIR/S125.

Coulbourn, W. 1981. Tectonics of the Nazca Plate and the continental margin of Western South America $18^{\circ}$ to $23^{\circ} \mathrm{S}$. Geol. Soc. America, Inc. Memoir, 154 : 587-681.

Craddock, J. y G. Mead. 1970. Midwater fishes from the Eastern South Pacific Ocean. Anton Bruun Rep. 3, Sci. Res. Southeast Pacific Exped., USA, 46 p.
De Buen, F. 1959a. Notas preliminares sobre la fauna marina preabismal de Chile, con descripción de una familia de rayas, dos géneros y siete especies nuevas. Biol. Mus. Nac. Hist. Nat., Santiago, 27(3): 173-201.

De Buen, F. 1959b. Lampreas, tiburones, rayas y peces de la Estación de Biología Marina de Montemar, Chile. Rev. Biol. Mar., Valparaíso, 9(1-3): 1-200.

De Buen, F. 1961a. Peces chilenos: familias Alepocephalidae, Muraenidae, Sciaenidae, Scorpaenidae, Liparidae y Bothidae. Rev. Biol. Mar., Valparaíso, 11(1): 1-52.

De Buen, F. 1961b. Resultados de una campaña ictiológica en las provincias del norte. Zonárida, 4: $1-4$.

Domínguez, P. y L. Zúñiga. 1976. Análisis fenológico de los cladóceros limnéticos (Crustácea: Entomostraca) de la laguna El Plateado (Valparaíso). An. Mus. Hist. Nat., Valparaíso, 9: 35-44.

Ebeling, A.W. 1962. Melamphaeidae I. Systematics and zoogeography of the species in the bathypelagic fish genus Melamphaes Günther. Dana Rep., 58: 1-164.

Ebeling, A. y H. Weed. 1963. Melamphaeidae III. Systematics and distribution of the species in the bathypelagic fish genus Scopelogadus Vaillant. Dana Rep., 60: 1-58.

Fisher, R. y R. Raitt. 1962. Topography and structure of the Perú-Chile Trench. Deep-Sea Res., 5(9): 423 443.

Fowler, H. 1951. Analysis of the fishes of Chile. Rev. Chil. Hist. Nat., 51-53: 263-326.

Fuenzalida, R. 1990. Variabilidad temporal de un índice de surgencia para la zona de Iquique (Lat. $\left.20^{\circ} \mathrm{S}\right)$. Invest. Cient. Y Tec., Serie Ciencias del Mar, UNAP 1: 37-47.

Fuenzalida, R. 1993. Prospección de recursos bentodemersales: condiciones oceanográficas. In: Aplicación de nuevas tecnologías para la captura de recursos hidrobiológicos marinos no tradicionales en la I Región. W. Palma (Ed.) Proyecto CHI/ 87/007, PNUD/UNAP, Iquique, Chile, 120 p.

Garman, S. 1899. Reports on an exploration off the West coasts of Mexico, Central and South America and off the Galapagos Islands. Mem. Mus. Comp. Zool. Harvard, Coll., (24): 1-431.

Günther, A. 1887. Report on the deep-sea fishes 
collected by H.M.S. "Challenger" during years 1873-1876. Challenger Rep., 22(57): 1-268.

Herrera, G. 1993. Prospección de recursos bentodemersales: aspectos reproductivos. In: Aplicación de nuevas tecnologías para la captura de recursos hidrobiológicos marinos no tradicionales en la Primera Región. W. Palma (Ed.), Proyecto CHI/87/007, PNUD/UNAP, Iquique, Chile, 120 p.

Hildebrand, S. 1946. A descriptive catalog of the shore fishes of Perú. Bull. U.S. Nat. Mus., 189: 1-529.

Iwamoto, T. 1978. Eastern Pacific macrourids of the genus Coelorhynchus Giorna (Pisces, Gadiformes) with a description of a new species from Chile. Proc. Calif. Acad. Sci., 41(12): 307-337.

Iwamoto, T. 1979. Eastern Pacific macrourine grenadiers with seven branchiostegal rays (Pisces: Macrouridae). Proc. Calif. Acad. Sci., 42(5): 135179.

Iwamoto, T y Y. Sazonov. 1988. A review of the Southeastern Pacific Coryphaenoides (sensu lato) (Pisces, Gadiformes, Macrouridae). Proc. Calif. Acad. Sci., 45(3): 35-82.

Kato, S., S. Springer y M. Wagner. 1967. Field Guide to Eastern Pacific and Hawaiian Sharks. U.S. Fish. Wildl. Service. Circ, 271, 47 pp.

Kong, I. y J. Valdés. 1990. Sciaenidos de Chile: Análisis Taxonómico y Morfológico. Estud. Oceanol., 9: 13-56.

Kong, I. y R. Meléndez. 1991. Estudio taxonómico y sistemático de la ictiofauna de aguas profundas capturada entre Arica e Isla Mocha (18³0'38³0'S). Estud. Oceanol., 10: 1-81.

Kong, I., R. Meléndez y G. Henríquez. 1998. Los peces Ophidiiformes de aguas profundas entre Arica e Isla Mocha. Estud. Oceanol., 7: 1-15.

Lönnberg, E. 1905. The fishes of the Swedish South Polar Expedition. Wissenchaftliche Ergebnisse Swedische Südpolar-Expedition, 5(6): 1-69.

Mann, G. 1954. La vida de los peces en aguas chilenas. Instituto de Investigaciones Veterinarias y Universidad de Chile, Santiago, 343 pp.

Martínez, E. 1976. Variabilidad de los caracteres merísticos en Merluccius gayi (Guichenot, 1848) en la costa de Chile. Rev. Biol. Mar., Valparaíso, 16(1): 71-93.
Massay, S. 1983. Revisión de la lista de los peces marinos del Ecuador. Bol. Científico y Técnico, Instituto Nacional de Pesca, Guayaquil, Ecuador, 6(1): 1-113.

Meléndez, R. y D. Meneses. 1989. Tiburones del talud continental recolectados entre Arica (18 $19^{\circ}$ 'S) e isla Mocha (38³0'S), Chile. Invest. Mar., Valparaíso, 17: 3-73.

Menni, R y A. Gosztonyi. 1982. Benthic and semidemersal fish associations in the Argentine Sea. Stud. Neotr. Fauna and Env., 17: 1-29.

Moreno, C., W. Duarte y J. Zamorano. 1979. Variación latitudinal del número de especies de peces en el sublitoral rocoso: una explicación ecológica. Arch. Biol. Med. Exper., 12: 169-178.

Morillas, J. y F. Carrasco. 1976. Nuevo registro de Nemichthys scolopaceus Richardson (Pisces, Apodes, Nemichthyidae) en la costa de Chile. Bol. Soc. Biol. Concepción, 50: 123-127.

Navarro, R. 1984. Programa computacional para análisis numérico de comunidades: Diversidad y sobreposición. Medio Ambiente, 7: 82-87.

Núñez, L y J. Vásquez. 1987. Observaciones tróficas y de distribución espacial de peces asociados a un bosque submareal de Lessonia trabeculata. Estud. Oceanol., 6: 79-85.

Ojeda, P. 1983. Distribución latitudinal ybatimétrica de la ictiofauna demersal del extremo austral de Chile. Rev. Chil. Hist. Nat., 56: 61-70.

Palma, W. (Ed.). 1993. Aplicación de nuevas tecnologías para la captura de recursos hidrobiológicos marinos no tradicionales en la Primera Región. Proyecto CHI/87/007. PNUD/UNAP, Iquique, Chile. $120 \mathrm{pp}$.

Peden, A. 1975. Redescription and distribution of the rare deep-sea eel Xenomystax atrarius in the Eastern Pacific Ocean. J. Fish Res. Bd. Canada, 29: 1-12.

Pequeño, G. 1971. Sinopsis de Macrouriformes de Chile. Bol. Mus. Nac. Hist. Nat., Chile, 32: 269 298.

Pequeño, G. 1975. Peces del crucero Merluza V efectuado con el B/C "Carlos Darwin" entre Corral y Coquimbo. Enero-Febrero de 1970. Bol. Mus. Nac. Hist. Nat., Chile, 34: 227-232.

Pequeño, G. 1977. Colecciones chilenas de peces. I Catálogo de los peces marinos de la Universidad 
Austral de Chile. An. Mus. Hist. Nat. Valparaíso, 10: 75-94.

Pequeño, G. 1986. Nuevos zoárcidos para Chile y clave para géneros de la región (Pisces, Osteichthyes). Bol. Soc. Biol Concepción, 57: 183-188.

Pequeño, G. 1989. Peces de Chile. Lista Sistemática revisada y comentada. Rev. Biol. Mar. Valparaíso, 24(2): 1-132.

Pequeño, G. y J. Lamilla. 1993. Batoideos comunes a las costas de Chile y Argentina-Uruguay (Pisces: Chondrichthyes). Rev. Biol. Mar., Valparaíso, 28(2): 203-217.

Pequeño, G y G. Revuelta. 1980. Notacanthiform fishes en the continental slope of Chile. Copeia, 1980(4): 892-895.

Pozzi, A. y L. Bordale. 1935. Cuadro sistemático de los peces marinos de la República Argentina. Anal. Soc. Cient. Argentina, 4(120): 145-200.

Sáiz, F. 1975. Aspectos mesofaunísticos hipogeos en el Parque Nacional "Fray Jorge". I parte. Análisis comunitario. An. Mus. Hist. Nat., Valparaíso, 8: 2950 .

Sáiz, F. 1980. Experiencias en el uso de criterios de similitud en el estudio de comunidades. Arch. Biol. Med. Exp., 13: 387-402.

Sazonov, Y. y T. Iwamoto. 1992. Grenadiers (Pisces, Gadiformes) of the Nazca and Sala y Gómez Ridges, Southeastern Pacific. Proc. Calif. Acad. Sci., 48(2): 27-95.

Schweller, W., L. Kulm y R. Prince. 1984. Tectonics, structure and sedimentary framework of the PerúChile Trench. Geol. Soc. America, Inc. Memoir, 36: 1-111.

Sielfeld, W. y M. Vargas. 1992. Tiburones y rayas del Pacífico Sur Oriental. Publ. Depto. Ciencias del Mar, Univ. Arturo Prat, Iquique, 135 p.

Sielfeld, W., M. Vargas y R. Fuenzalida. 1995. Peces mesopelágicos frente a la costa norte de Chile (18 $25^{\prime}$-21 ${ }^{\circ} 47^{\prime}$ S ). Invest. Mar., Valparaíso, 23: 8397.

Recibido el 20 de Julio de 1995.

Aceptado el 8 de noviembre de 1996.
Sievers, H. y N. Silva. 1975. Masas de agua y circulación en el Océano Pacífico Suroriental $\left(18^{\circ}-33^{\circ} \mathrm{S}\right)$ (Operación Oceanográfica MARCHILE VII). Cienc. Y Tec. Mar. CONA, 1: 7-67.

Sievers, H. y N. Silva. 1982. Masas de agua y circulación geostrófica frente a la costa de Chile $\left(18^{\circ}-33^{\circ} \mathrm{S}\right)$ (Operación Oceanográfica MARCHILE VII). Cienc. y Tec. Mar, CONA, 6: 61-99.

Solervicens, J. 1973. Coleópteros del bosque de Quintero. An. Mus. Hist. Nat. Valparaíso, 6: 115-159.

Springer, S. 1979. A revision of the cat sharks, family Scyliorhinidae. NOAA Tech. Rep., NMFS, Circ., 422: 1-97.

Targett, T. 1981. Trophic ecology and structure of coastal Antarctic fish communities. Mar. Ecol. Progr. Ser., 4: 243-263.

Varas, E. y P. Ojeda. 1990. Intertidal fish assemblages of the Central Chilean coast: diversity, abundante and trophic patterns. Rev. Biol. Mar. Valparaíso, 25: 59-70.

Vargas, M. y W. Sielfeld. 1992. Bathyraja peruana McEachran y Miyake, 1984, nuevo registro para la condrictiofauna chilena. Invest. Cient. y Tec., Serie Ciencias del Mar, UNAP, 2: 105-110.

Vásquez, J. 1989. Estructura y organización de huirales submareales de Lessonia trabeculata. Tesis Doctoral, Facultad de Ciencias, Universidad de Chile, $261 \mathrm{p}$.

Yáñez, E. y M.A. Barbieri. 1983. Aplicación de métodos de inercia. Estudio de la repartción de la fauna bento-demersal en la región de Valparaíso (33으$72^{\circ} \mathrm{W}$ ). Análisis de la estructura del langostino colorado (Pleuroncodes monodon) en la zona central de Chile. In: Recursos Marinos del Pacífico. P. Arana (Ed.), U.S. Inter. Sea Grant Program y Escuela de Ciencias del Mar, UCV, Valparaíso: 295-319.

Zama, A. y E. Cárdenas. 1984. Descriptive catalogue of marine and freshwater fishes from the Aysén Region, Southern Chile, with zoogeographical notes on the fish fauna. Jap. Int. Coop. Ag. (JICA), Rep., 9: 1-75. 


\section{Anexo 1. Cuadro sistemático y distribución latitudinal de las especies capturadas. Annex 1. Systematic description and latitudinal distribution of the species captured.}

\section{Clase Chondrichthyes \\ Orden Squaliformes}

Familia Squalidae

Aculeola nigra De Buen, 1959: Trujillo-Perú (Compagno, 1984b) a Concepción (De Buen, 1959a)

Centroscyllium granulatum Günther, 1887:

Malvinas (Günther, 1887) hasta 3003'S;

(Meléndez y Meneses, 1989; y presentes datos).

Centroscyllium nigrum Garman, 1889: Panamá (Garman, 1899) a 38¹1'S (Meléndez y Meneses, 1989).

Familia Scyliorhinidae

Cephalurus sp.: Baja California (Springer, 1979)

a Coquimbo (29º ) (Meléndez y Meneses, 1989).

Familia Rajidae

Bathyraja peruana McEachran y Miyake, 1984: Ecuador ( $\left.3^{\circ} \mathrm{S}\right)$ a $21^{\circ} 26^{\prime} \mathrm{S}$ (Vargas y Sielfeld, 1992). Sympterygia brevicaudata (Cope, 1877): Ecuador $\left(3^{\circ} \mathrm{S}\right)$ a Chile Central $\left(37^{\circ} \mathrm{S}\right)$ (Sielfeld y Vargas, 1992).

Familia Pseudorajidae

Gurgesiella furvescens De Buen, 1959: Galápagos a Valparaíso (32응 (Sielfeld y Vargas, 1992).

Familia Myliobatidae

Myliobatis peruvianus Garman, 1913: Paita-Perú (Chirichigno, 1974) a Valdivia (Pequeño, 1977).

\section{Clase Holocephali}

\section{Orden Chimaeriformes}

Familia Chimaeridae

Hydrolagus macropthalmus De Buen, 1959: Valparaíso (De Buen, 1959a) a Perú (Chirichigno, 1974).

\section{Clase Osteichthyes \\ Orden Anguilliformes}

Familia Congridae

Xenomystax atrarius Gilbert, 1891: Vancouver-Canadá (Peden, 1975) a Valparaíso (Kong y Meléndez, 1991).

Familia Ophichthyidae

Ophichthus ater Peters, 1866: Iquique (Fowler, 1951).
Familia Nemichthyidae

Nemichthys scolopaceus Richardson, 1848: Concepción (Morillas y Carrasco, 1976) al norte: circumglobal.

\section{Orden Notacanthiformes}

Familia Notacanthidae

Notacanthus sexpinnis Richardson, 1846: amplia distribución en el Pacífico, Indico y Atlántico (Pequeño y Revuelta, 1980) .

Familia Halosauridae

Halosaurus radiatus Garman, 1899: Golfo de $\mathrm{Pa}-$ namá (Garman, 1899) a Pichilemu (Pequeño y Revuelta, 1980).

\section{Orden Salmoniformes}

Familia Alepocephalidae

Binghamnichthys microphos (Parr, 1937): cita para Chile sin comentario de Pequeño (1989).

Alepocephalus sp. 1: 19 $51^{\prime}-21^{\circ} 20^{\prime} \mathrm{S}$ del presente estudio.

Roulenia sp. 1: 2046'S del presente estudio.

\section{Orden Batrachoidiformes}

Familia Batrachoididae

Aphos porosus (Valenciennes, 1837): Puerto Pizarro-Perú (De Buen, 1959b) a sector norte XII Región (Lönnberg, 1905).

\section{Orden Lophiiformes}

Familia Ogcocephalidae

Dibranchus spinosa (Garman, 1899): $7-8^{\circ} \mathrm{N}$ (Garman, 1899; Bussing 1965) a 2047's (estaciones 6 y 11 presente estudio).

\section{Orden Gadiformes}

Familia Merlucciidae

Merluccius gayi (Guichenot, 1848): $47^{\circ} \mathrm{S}$ (Martínez, 1976) a Ecuador (Massay, 1983).

Familia Macrouridae

Coryphaenoides ariommus Gilbert y Thomson, 1916: norte de Perú $\left(4^{\circ} \mathrm{S}\right)$ a sur de Chile $\left(38^{\circ} \mathrm{S}\right)$ (Iwamoto y Sazonov, 1988). 
Coryphaenoides delsolari Chirichigno y Iwamoto: Islas Galápagos (Chirichigno y Iwamoto, 1977) a 3543'S (Kong y Meléndez, 1991).

Coryphaenoides anguliceps $($ Garman, 1899) $=\sin$. Lyraticeps Garman, 1899: México a Perú (Iwamoto y Sazonov, 1988); 26²46’S (estación 6 del presente estudio).

Nezumia pulchella (Pequeño, 1971): $7^{\circ} 49^{\prime}$ S a $33^{\circ} \mathrm{S}$ (Iwamoto, 1979).

Trachyrhynchus helolepis Gilbert, 1891: Columbia Británica (Iwamoto, 1979) a $26^{\circ} \mathrm{S}$ (Kong y Meléndez, 1991).

Trachyrhynchus Villegai Pequeño, 1971: Norte de Perú (Chirichigno, 1974) a $30^{\circ}$ S (Pequeño, 1971).

\section{Orden Ophidiiformes}

Familia Ophidiidae

Cherublemma emmelas (Gilbert, 1890): 14²27' N a 849'S (Chirichigno, 1974); 19¹9'S (Kong et al., 1988) y $21^{\circ} 25^{\prime} \mathrm{S}$ (estación 2 del presente estudio).

Dicrolene nigra Garman, 1899: Golfo de Panamá a 18²3'S (Chirichigno, 1974); 18-27³6's (Kong et al., 1988).

Monomitopus torvus Garman, 1899: 6 $30^{\circ}-7^{\circ} 30^{\prime} \mathrm{N}$ (Garman, 1899); 18²6'S-3407'S (Kong et al., 1988).

Cataetyx rubrirostris Gilbert, 1890: costas de

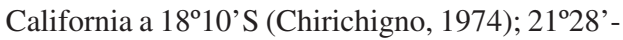
$35^{\circ} 38^{\prime}$ 'S (Kong et al., 1988).

Cataetyx simus Garman, 1899: Golfo de Panamá a 35³2'S (Kong et al., 1988).

\section{Orden Beryciformes}

Familia Trachichthyidae

Trachichthys mento Garman, 1899: 7²21'N (Garman, 1899) a 36²8’'S (Kong et al., 1988).

Familia Melamphaeidae

Melamphaes acanthomus Ebeling, 1962: sur de California a Golfo de Panamá (Ebeling, 1962); el presente estudio hasta $19^{\circ} 45^{\prime} \mathrm{S}$ (estación 11 del presente estudio).

Scopelogadus mizolepis (Günther, 1878): océanos Pacífico, Atlántico e Indico tropicales (Ebeling y Weed, 1963); hasta $34^{\circ} \mathrm{S}$ (Craddock y Mead, 1970).

\section{Orden Scorpaeniformes}

Familia Psychrolutidae

Psychrolutes sio Nelson, 1980: Punta PicosPerú a Valparaíso (Kong y Meléndez, 1991).

Familia Normanichtyidae

Normanichthys crookeri Clark, 1937: 55³0’S en el Atlántico argentino (Pozzi y Bordale, 1935); Aysén: 45¹0'S (Zama y Cardenas, 1984) hasta Perú-Chimbote (Chirichigno, 1974).

\section{Orden Perciformes}

Familia Serranidae

Paralabrax humeralis (Valenciennes, 1828): Puerto Pizarro-Perú al sur de Chile (Chirichigno, 1974).

Familia Pomadasyidae

Isacia conceptionis (Cuvier, 1830): Isla Lobos de Afuera-Perú a Talcahuano-Chile (Chirichigno, 1974); probablemente al menos hasta Valdivia.

Familia Sciaenidae

Sciaena deliciosa (Tschudi, 1844): Puerto Pizarro-Perú a Corral (Kong y Valdés, 1990).

Familia Centrolophidae

Seriolella violacea Guichenot, 1848: TalaraPerú a Valparaíso (Chirichigno, 1974).

Familia Zoarcidae

Bothrocara alalonga (Garman, 1899): 27³4'N (Garman, 1899) a 2047'S (estación 6, presente estudio).

Pachychara suspectum (Garman, 1899): Golfo de California a Golfo de Panamá (Anderson y Peden, 1988); $18^{\circ} 25^{\prime}$ S (estación 15, presente estudio).

Pachychara sp. 1:2046'S del presente estudio.

Familia Trichiuridae

Aphanopus carbo Lowe, 1839: Especie pelágica de aguas profundas del Atlántico oriental y Pacífico sur oriental (Kong y Meléndez, 1991).

\section{Orden Pleuronectiformes}

Familia Bothidae

Hippoglossina macrops Steindachner, 1876: desde Mazatlán-México (Chirichigno, 1974) a Aysén (46²16'S) (Zama y Cardenas, 1984). 